\title{
Parametric investigation of boundary layer control using triangular micro vortex generators
}

\author{
Milad Bagheri ${ }^{1, \mathrm{a}}$, Motassem Al Muslmani ${ }^{1}$, Asad Masood ${ }^{1}$, Mahmood Khosravi ${ }^{1}$, Mohamed Atef Mahmoud ${ }^{1}$, Aniket \\ Cardoz ${ }^{1}$, Abdulrahman Akkuwari ${ }^{1}$, Yusuf Alanezi ${ }^{1}$, and Young Kim ${ }^{2}$. \\ ${ }^{1}$ Aerodynamics, Dept of Aeronautical Engineering, Emirates Aviation College, Dubai, UAE \\ ${ }^{2}$ Assistant professor, Dept, of Aeronautical Engineering, P.O. Box 53044, Emirates Aviation College, Dubai, UAE
}

\begin{abstract}
Improving the aerodynamic performance of an airfoil is one of the primary interests of the Aerodynamicists. Such performance improvement can be achieved using passive or active flow control devices. One of such passive devices having a compact size along with an effective performance is the Micro Vortex Generators (MVGs). A special type of MVGs, which has been recently introduced in the aerospace industry, is "Triangular Shape" MVGs and its impact on aerodynamic characteristics is the main interest of this study. This study will compare the effects of various configurations through which delay of the flow separation using boundary layer control will be analysed by experimental and theoretical approach. The experimental investigations have been conducted using subsonic wind tunnel and the theoretical analysis using ANSYS ${ }^{\circledR}$ 13.0 FLUENT of which the final results are compared with each other.
\end{abstract}

\section{Introduction}

Engineers around the world are constantly refining, developing and experimenting new methods in improving aircraft performance. One of such flight performance enhancing device is the Triangular MVGs. These are passive flow control devices which can be placed on different location on the aircrafts such as wings leading edge, fuselage surface, and engine nacelles. Doing so can delay flow separation at low speeds and improve flight control. Generally aerospace industry is not the only place which can benefit from these devices. Triangular MVGs can be applied to various engineering fields such as automobile and marine industries.

There are a number of unique advantages of these vortex generators. First of all, these types of vortex generators are compact in size which does not add extra drag and furthermore it is easy to install on various locations on the aircraft surfaces [2,3]. Other remarkable advantages of MVGs are noise suppression, reduced fuel consumption and reduced skin friction drag by delaying the laminar-turbulent transition. This also helps in aircraft to extend the range and improves longitudinal manoeuvrability.

For this project it was required to investigate aerodynamic characteristics of triangular MVGs when placed on an aluminium flat plate. In order to do so, subsonic wind tunnel experiments as well as theoretical computation using ANSYS were conducted, to identify the impact and outcomes of various dimensions of

\footnotetext{
${ }^{\mathrm{a}}$ Milad Bagheri: milad.br@live.com
}

triangular MVGs and to identify the best triangular configuration with best aerodynamic performance.

\section{Motivation}

Figure 1 depicts an example of the triangular MVGs which are being used on the wing leading edge of Columbia 400. The size of the MVGs can be noticed by reference to the finger. Such triangular MVGs can easily be applied on existing aircrafts. This privilege of using triangular MVGs develops great interest in investigating the properties and aerodynamics effects of such MVGs on aircraft performance.

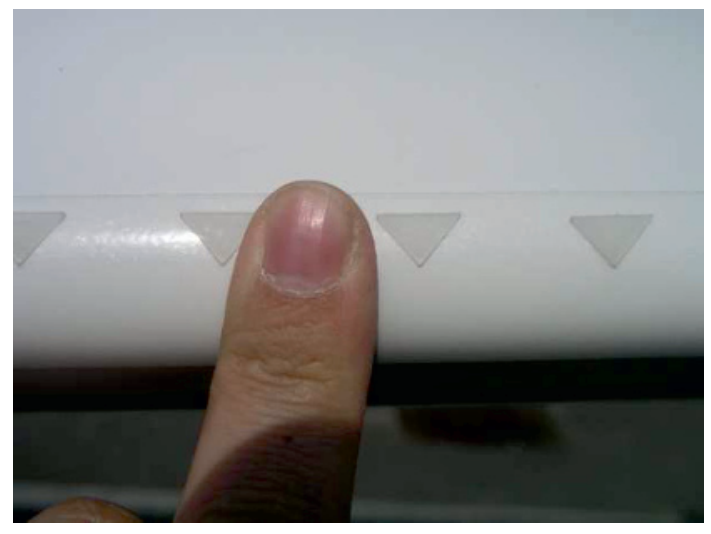

Figure 1. TMVGs on leading edge of wing of Columbia 400 


\section{Background Information}

As air normally flows over the wing of an aircraft during flight the air "sticks" to the surface of the wing. This adherence to the wing's surface produces lift. If the airflow loses its adherence and separates from the wing, aircraft performance can suffer in the form of increased drag, loss of lift and higher fuel consumption.Vortex generators are an external aerodynamic surface applied to different types of aircrafts to counter this drop in performance. Vortex generators can be placed in various locations such as wings and engine nacelles and can be found in more than one shape. This project is centred on Micro Vortex Generators which have a superior effect than Vortex generators on the enhancing aircraft performance since the drag produced by MVGs is less than VGs.

\section{Working Principles}

As mentioned in introduction, MVGs can be placed in various locations; the main purpose of applying these devices is creating longitudinal vortices which force the airflow to remain attached to surface, this results in the delay of flow separation and improvement in stall characteristics by increasing stall angle of attack which subsequently causes an increase in lift coefficient.

Figure 2 shows a comparison between an airfoil before and after application of MVGs, this illustration shows the airflow direction and effectiveness of MVGs in attaching airflow to surfaces, as discussed in the introduction, this results in reduction of wake which is a region of recirculating flow.

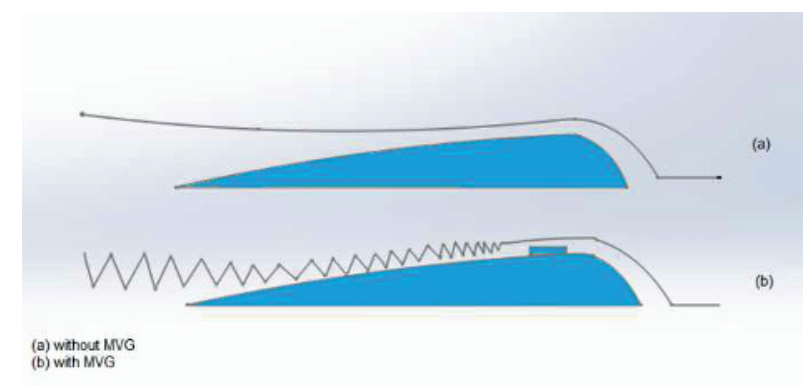

Figure 2. Working Principle of Micro Vortex Generators.

\section{Theory}

\subsection{Boundary layer}

When fluid flows pass through an immersed body, a thin boundary layer will be developed near the solid body due to the no-slip condition. The flow can be treated as inviscid flow outside of this boundary layer, while viscous effects are important inside of this boundary layer. As the flow moves along the flat plate there is a change of flow in the chordwise direction from laminar to turbulent. The height of the boundary layer needs to be measured as well as the behaviour of the flow as the height increases. This is done in order to place the MVGs within the laminar region. It is placed in the laminar region to avoid the mixing of turbulent and laminar flow and intern to cause less drag.

\subsection{Wall pressure fluctuations downstream of a rearward facing step}

The effect of flow over a backward facing step can be explained as an irregular boundary-layer flow over any surface which has a certain amount of height and for the downstream surface is less compared to that of the upstream surface. This effect involves the upstream flow of the turbulent boundary layer to separate at the irregularity of the surface and the area exactly at the irregularity, a recirculation in a local region is formed which also forms a free shear layer. This recirculation region is the vortex generated by the MVGs and causes the delay of flow separation.

\section{Wind Tunnel Test}

Low Speed Wind tunnel experiment was carried out using a 2-D aluminium flat plate with the length of 400 $\mathrm{mm}$, width of $300 \mathrm{~mm}$, and thickness of $16 \mathrm{~mm}$. In order to determine the location of positioning the triangles, it was required to determine the following three objectives.

The first objective was to determine the constant pressure area on the flat plate to distinguish optimum location in order to locate MVGs. This objective has been achieved by measuring streamwise surface pressure distribution.

The second objective is to identify boundary layer thickness over the flat plate. The purpose is having MVGs placed within the boundary layer otherwise it would be ineffective. In order to measure the boundary layer thickness, it is required to measure the dynamic pressure from the surface upwards, either by using a transverse method or using wake rake. By applying the Bernoulli theory [1], the velocity change moving from the surface upwards can be measured. Starting from zero velocity on the surface (no slip condition theory) the velocity gradually increases up till reaching the free stream velocity. By measuring the point from zero velocity (surface) to the point after which velocity reaches freestream velocity the boundary layer thickness is measured.

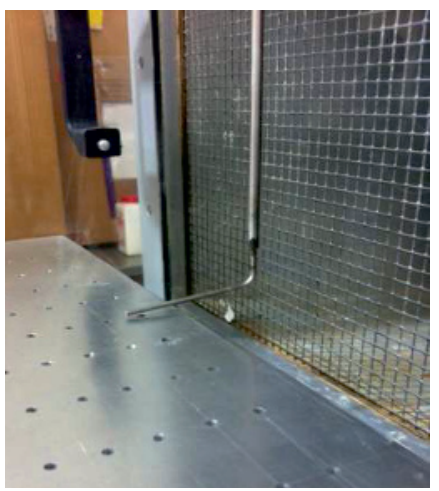

Figure 3. Boundary layer thickness measurement using transverse method 
Furthermore, the third and final objective was to determine the behaviour of flow over the flat plat. Since the micro vortex generators are to be placed in laminar flow for minimum drag, the pressure is required to be measured from the surface, upwards at various chord wise locations as shown in figure 4 .

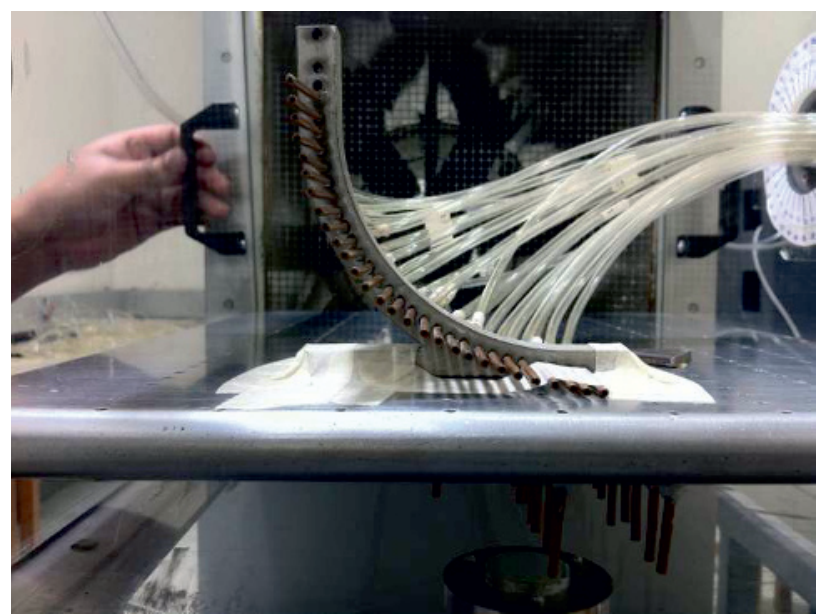

Figure 4. Boundary layer thickness measurement using wake rake

In order to achieve the three objectives prior to applying the TMVGs, experiments were conducted on the aluminium flat and the results are published in section 6.1. Once the optimum location of the TMVGs was determined, experiments were carried out to investigate the behaviour of MVGs. Various TMVGs configurations with different dimensions were used to differentiate the effects of each configuration on vortex strengths.

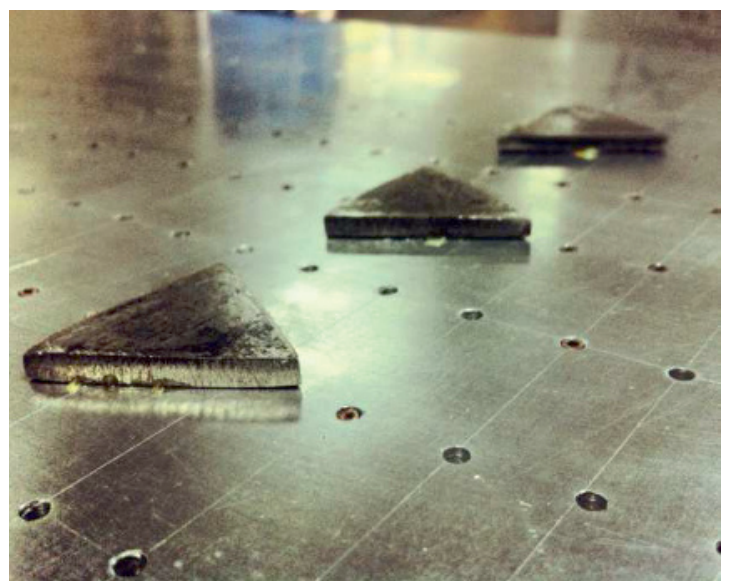

Figure 5.Triangualrs MVGs on aluminium flat plate

\subsection{Experimental Results}

A wake rake was developed as shown in figure 4 in order to calculate the flat plate boundary layer thickness that was found out to be $7 \mathrm{~mm}$ which indicates the allowable thickness of TMVGs.

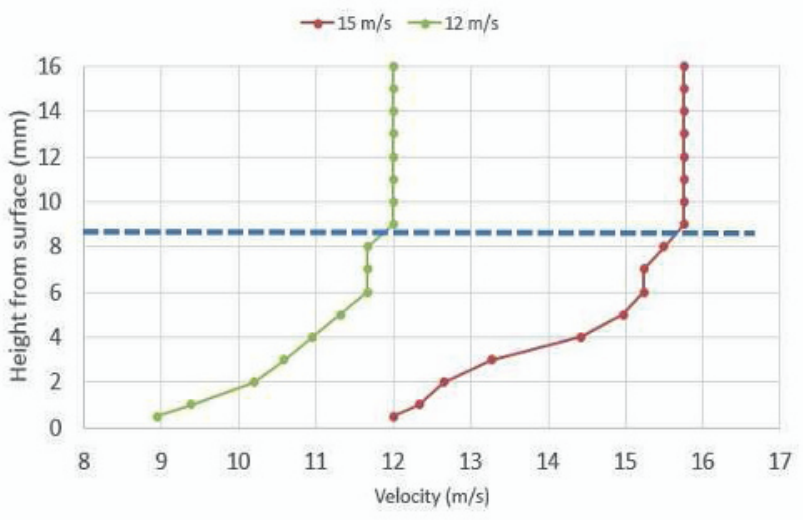

Figure 6. Boundary layer thickness at $15 \mathrm{~m} / \mathrm{s}$ and $12 \mathrm{~m} / \mathrm{s}$

Figure 6 portrays the results obtained from the wind tunnel experiment using the wake rake, in which the pressure was measured from the surface of the flat plate, upwards. The two lines in figure 6 represent two wind velocities at which the readings were recorded. Both lines seem to stabilize after crossing $8 \mathrm{~mm}$ mark. However the boundary layer was estimated to $7 \mathrm{~mm}$ as a safety measure. This was taken to be the boundary layer thickness over the flat plate on the aft section of the plate, indicating the allowable thickness of Triangular MVGs. However the thickness of the TMVGs was taken to be 4 $\mathrm{mm}$ as this will conform it presence within the boundary layer.

The next conducted experiment was the measurement of pressure coefficient in the streamwise direction and the results are shown in figure 7.

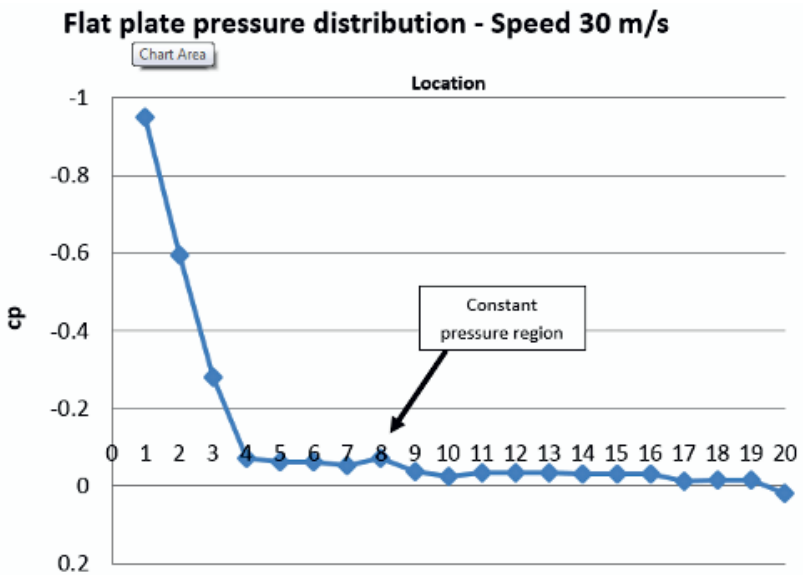

Figure 7. Pressure coefficient variation across the flat plate

Figure 7 shows surface pressure distribution based on wind tunnel test. Starting from the leading edge, 20 streamwise static pressure taps were arranged. Maximum pressure coefficient was recorded on tap 1 after which it decreases until tap 5 after which remains constant. The MVGs exact location was calculated to be at constant pressure region which was begins from tap 5 onwards i.e. $62.5 \mathrm{~mm}$ from LE.

The next experiment was conducted to determine the flow behaviour at the place of application on the triangles since it was required for the triangles to be placed in 
laminar region. The figure 8 shows the flow behaviour at the constant pressure region.

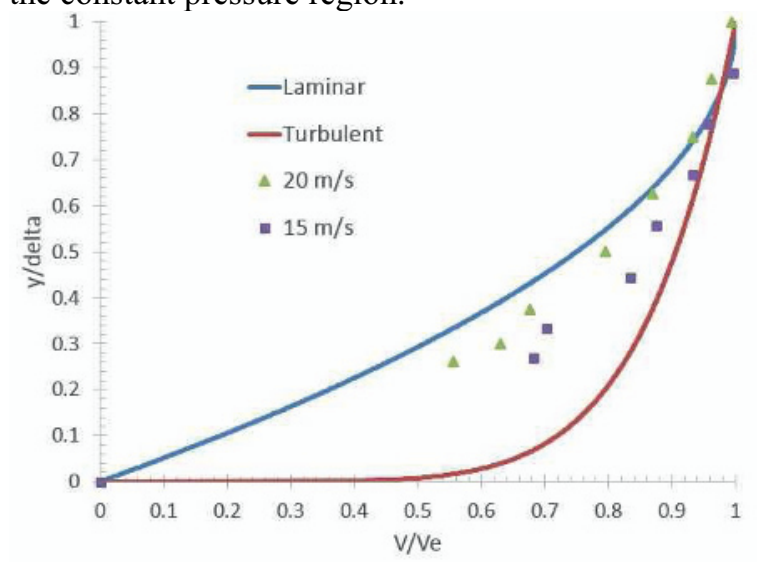

Figure 8. Pressure variation across the flat plate $-90 \mathrm{~mm}$ from LE

Figure 8 shows four lines, out of which the blue and red represent the theoretical pressure trend in laminar (blue) and turbulent (red) region respectively. The other two lines in green and purple represent pressure trend at 20 and $15 \mathrm{~m} / \mathrm{s}$ respectively. The wake rake was placed 90 $\mathrm{mm}$ away from the leading edge, within the constant pressure region. The trend to results using velocity 15 and 20 show a laminar flow, making it a suitable location to place the TMVGs.

The wake rake was later placed further away on the flat plate, at $300 \mathrm{~mm}$ from lead edge. Figure 9 shows the wind tunnel experiment results obtained at this chordwise position.

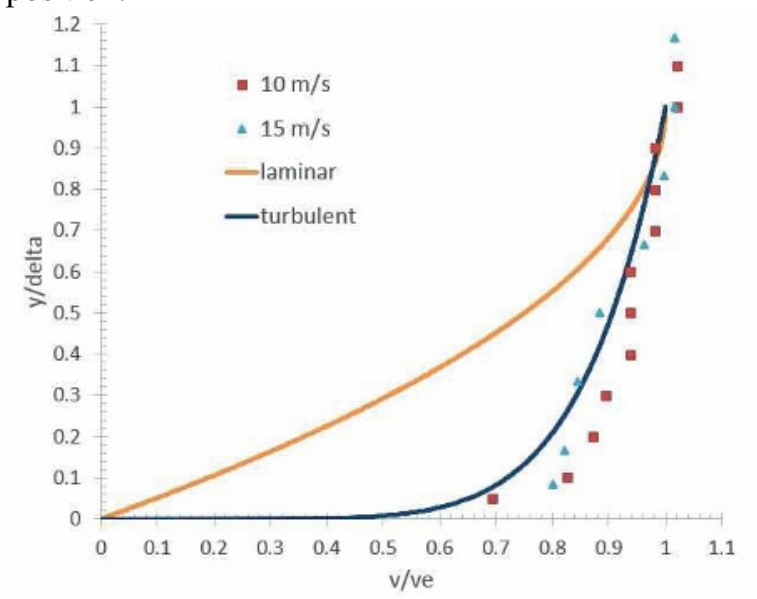

Figure 9. Pressure variation across the flat plate -300 $\mathrm{mm}$ from LE

The graph in figure 9 was obtained by running the wind tunnel on various speeds of 15 and 10 meter per second with the wake rake measuring pressure, placed $300 \mathrm{~mm}$ away from the leading edge onto the flat plate. Likewise the figure 8 , the graph (figure 9) consists of four lines, in which the green and blue represent laminar and turbulent flow lines obtained from boundary layer theory. Whereas the red and light blue line represents the experimental data obtained at speeds 10 and 15 . It can be observed from figure 9 that flow at this stage reached turbulent behaviour and thus is unsuitable for vortex formation.

By this stage the three objectives prior to placing the TMVGs are achieved using the above mentioned experiments. Analysing the results has provided an area on the flat plate with constant pressure and laminar flow. Moreover the boundary layer thickness over the flat plate of such laminar constant pressure reign was also calculated. Based on these results three triangles configuration were manufactures and placed over the flat plate for experimentation. Three configurations were manufactured as shown in Table 1.

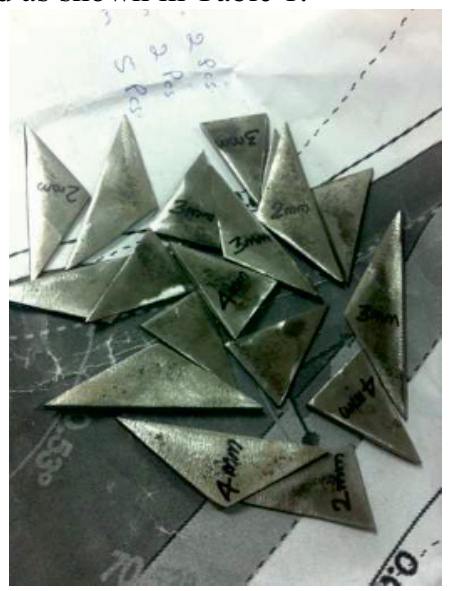

Figure 10. TMVGs of different configurations

Flat plat vs equalateral traingle pressure distribution graph - Speed 30 $\mathrm{m} / \mathrm{s}$

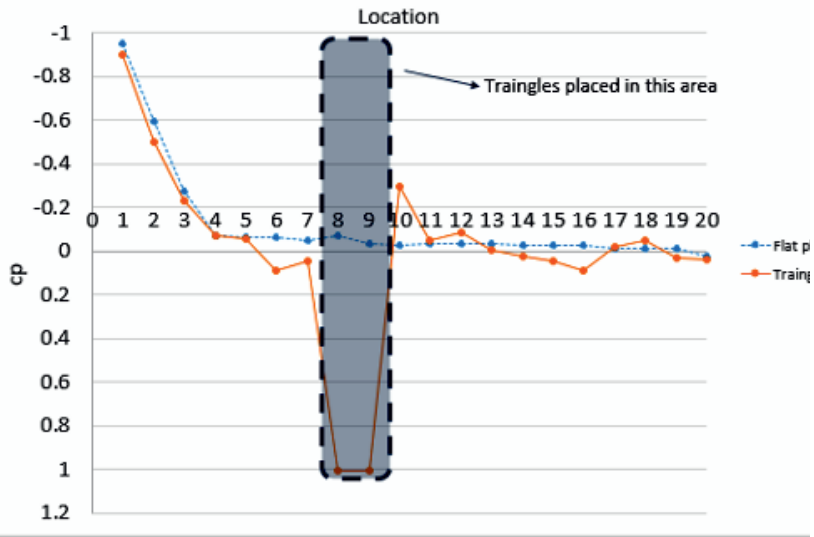

Figure 11. Variation of pressure coefficient in chordwise direction of flat plate

Figure 11 shows the dashed line (blue) representing the streamwise pressure distribution without MVGs, while the solid line (orange) presents the pressure distribution with MVGs. An equilateral triangle of thickness $4 \mathrm{~mm}$ was placed on the constant pressure region. Maximum pressure coefficient was recorded on tap 1 after which it decreases until tap 5 after which remains constant. Triangles were placed further with in the constant pressure reign as a safe precaution. Tap number 8 and 9, in case of MVGs application, display pressure coefficient of 1 as the pressure taps were 
covered by MVGs; thus also represents the location of triangles placement along the flat plate.

It can be observed from figure 11 , that there is a slight increase in pressure at tap number 6 and 7 representing conditions close to stagnation, as air approaches the leading edge of the triangular MVGs. while, tap 10 onwards, represent the pressure over the flat plate of the flow after crossing the triangular MVGs. A sudden drop in pressure at tap 10 represents flow circulation due to the backward facing step theory; and from tap 11 onwards the flow stabilizes and becomes constant. Similar results trends were observed on experiments conducted on other TMVGs configurations.

\subsection{Flow visualisation}

At this stage it is still unclear to say that vortices are even being formed. Thus in order to prove the formation of vortex, flow visualisation was conducted. Figure 12 shows the results obtained after conducting flow visualisation of the three TMVGs configurations. The vortex can be clearly seen being formed at the edges of each triangle. Visualization of the Created vortex on MVGs is difficult due to unsteady nature of the flow at the region where the vortex is created [4].

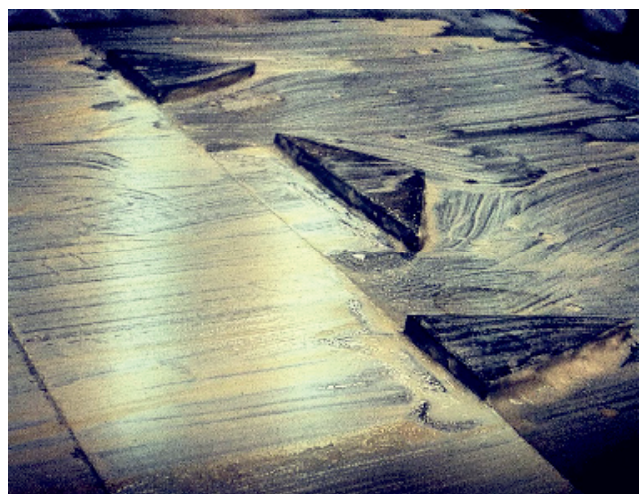

Figure 12. Flow visualisation results

\section{Computational Fluid Dynamics}

To validate obtained experimental results, ANSYS (CFD) engineering simulation software was used. The initial step was to model the flat plate using computer-aided design software. After this step, the model was imported to ANSYS ${ }^{\circledR} 13.0$ FLUENT ${ }^{\circledR}$.

The final configuration of the generated flat plate is shown in figure 13.

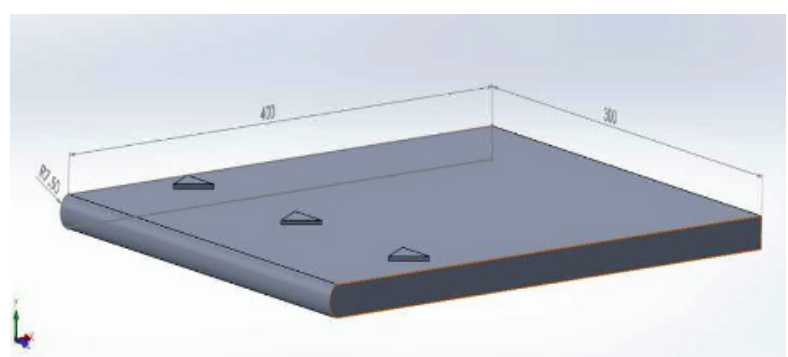

Figure 13. Triangular MVGs on aluminium flat plate (CAD Model)
Table 1.Three Different Configurations of MVGs.

\begin{tabular}{|c|c|c|}
\hline Shape & Design \\
\hline 1 & & \\
\hline 3 & & \\
\hline
\end{tabular}

Table 1 shows three Triangular MVGs configurations tested in wind tunnel along with ANSYS Fluent software.

To start analysis in ANSYS Fluent software, a virtual wind tunnel is constructed which simulates the experimental area of the model; this virtual wind tunnel is shown in figure 14 .

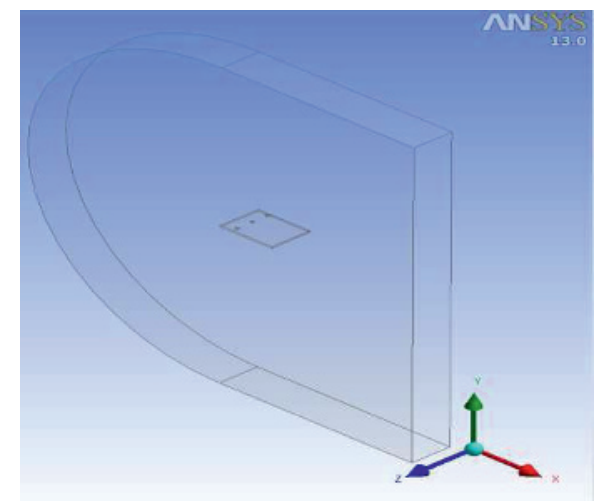

Figure 14. Virtual Wind Tunnel 


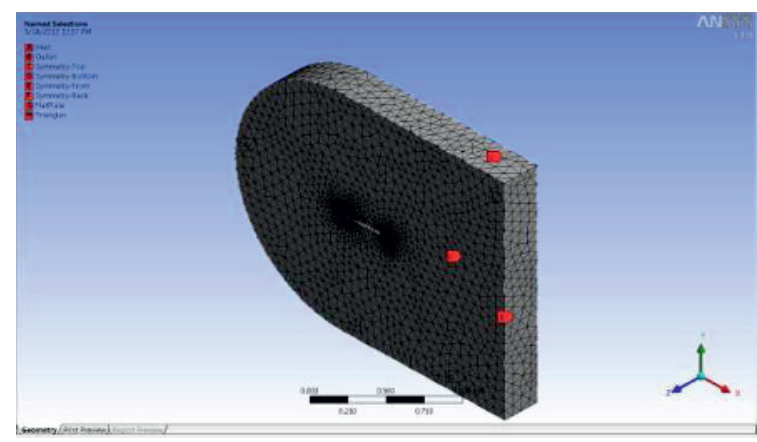

Figure 15. Virtual wind tunnel mesh structure

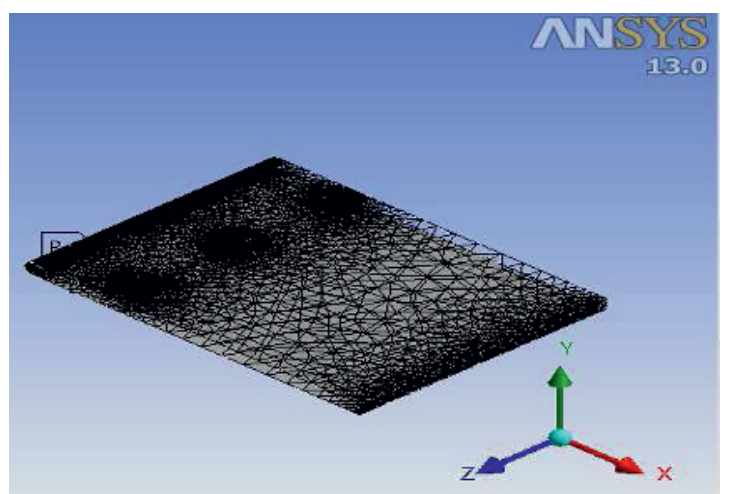

Figure 16. Flat plate mesh Structure

Based on figure 16 , the mesh structure is observed to be denser around the MVGs, to make sure ANSYS analysis creates detailed flow characteristics around the MVGs position. The main aim is to monitor flow field created when applying triangular MVGs, since the airflow is expected to have variation when flowing over and around the MVGs.

\subsection{CFD Results}

Using ANSYS the boundary layer thickness of the flat plate with free stream velocity of $30 \mathrm{~m} / \mathrm{s}$ without application of MVGs was found out to be $7.5 \mathrm{~mm}$ which matches the experimental results. This thickness is important to identify the MVGs thickness, as the main purpose is to keep the flow in the laminar region and to avoid laminar to turbulent transition region.

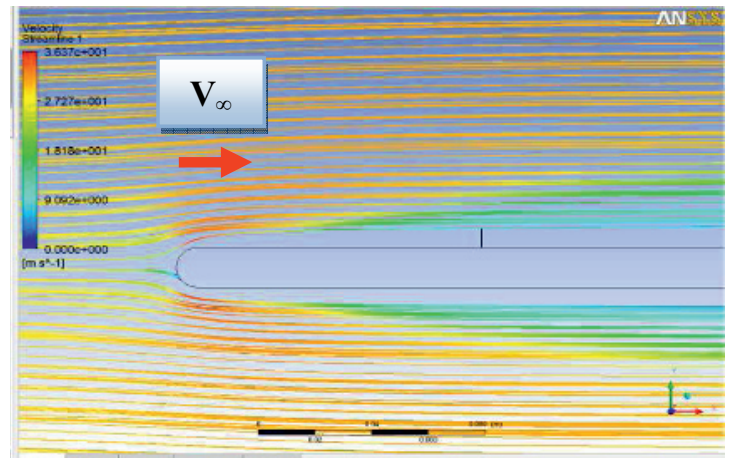

Figure 17. Boundary layer measurement
The main objective of ANSYS analysis is to ensure that TMVGs are capable of generating vortices (small tornadoes) that force the airflow to remain attached to a surface. The following results are obtained using ANSYS Fluent software.

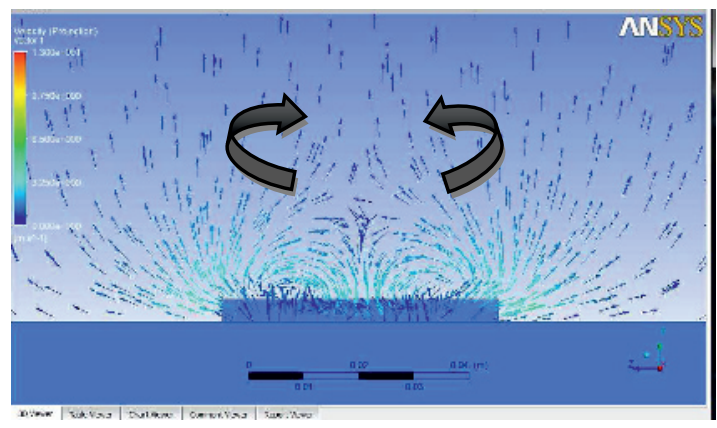

Figure 18. Vortices created by TMVGs (Back View).

As can be seen in Figure 18, it is apparent that Triangular MVGs create vortices. The vortex core region is displayed in Figure 18, having the velocity at the triangles tips to be around $9 \mathrm{~m} / \mathrm{s}$ decreasing to approximately $2 \mathrm{~m} / \mathrm{s}$ when moving towards MVGs center.

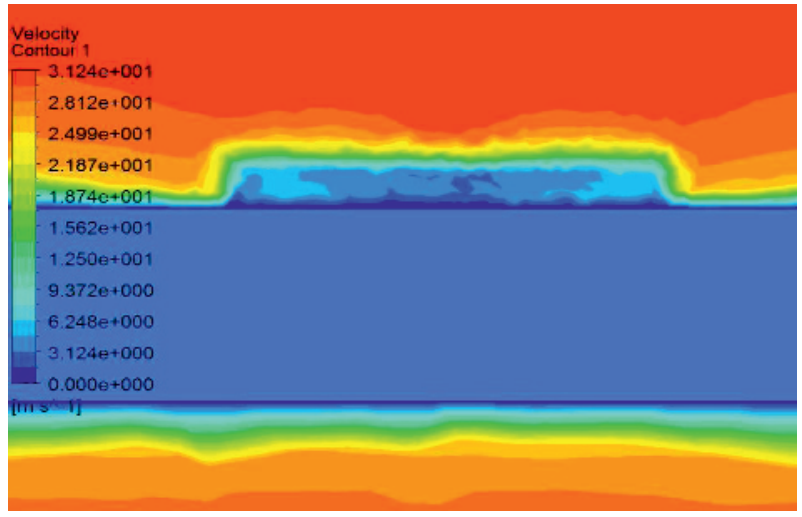

Figure 19. Velocity Contour (Back View).

Figure 19 assists the better understanding of flow field structure. It can be observed that local flow velocity is $30 \mathrm{~m} / \mathrm{s}$ and due to the no slip condition, the velocity at the surface is zero and this velocity increases by moving toward the top surface of MVG.

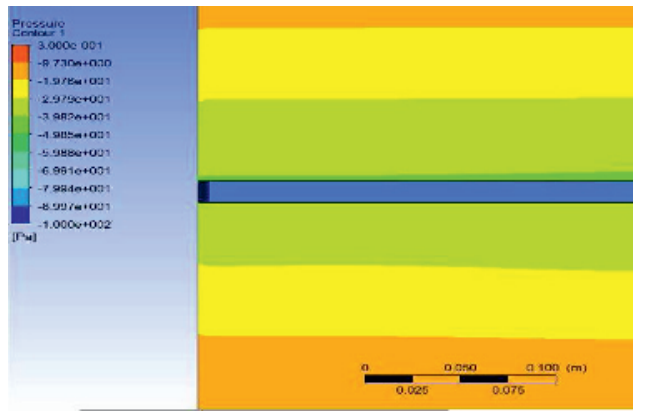

Figure 20. Pressure Contour on flat plate (Back View). 


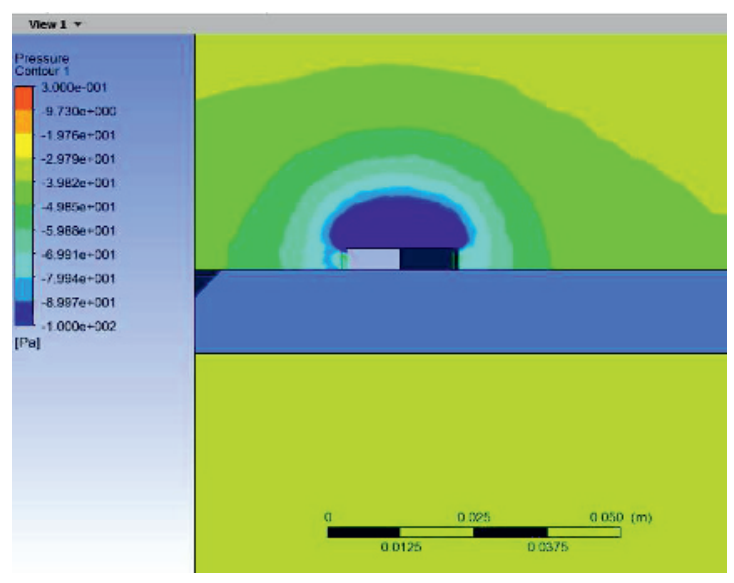

Figure 21. Pressure Contour on flat plate with MVGs (Back View).

Figures 20 and 21 illustrate comparison in static pressure after and before application of TMVGs. As can be seen, the pressure remains constant on the flat plate surface. While after application of MVGs, the pressure is least on top of the MVGs surface, nearby to the axis, and intensify as it moves away from the core of MVGs, according to Bernoulli's Principle, therefore the pressure gradient forces the air to curve around the axis.

Table 2. Velocity streamlines showing vorticity of three different MVGs.

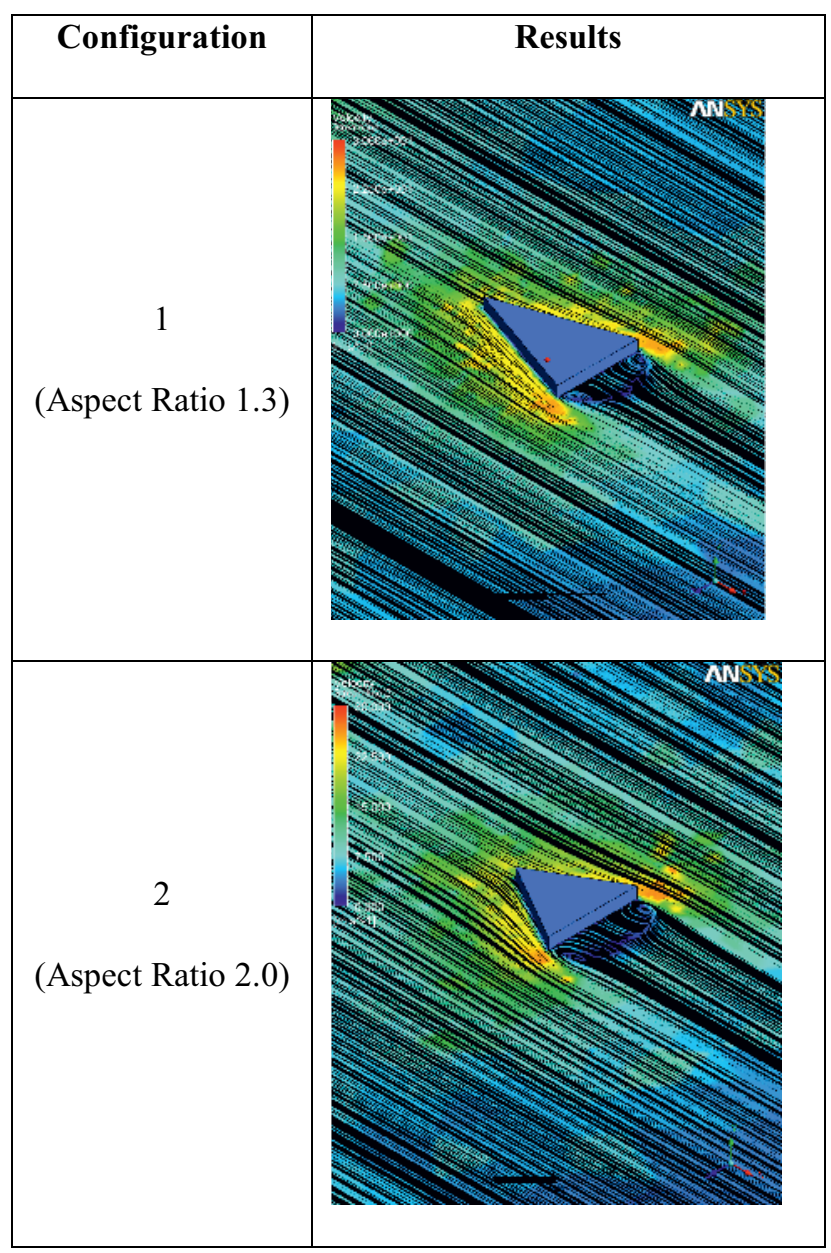

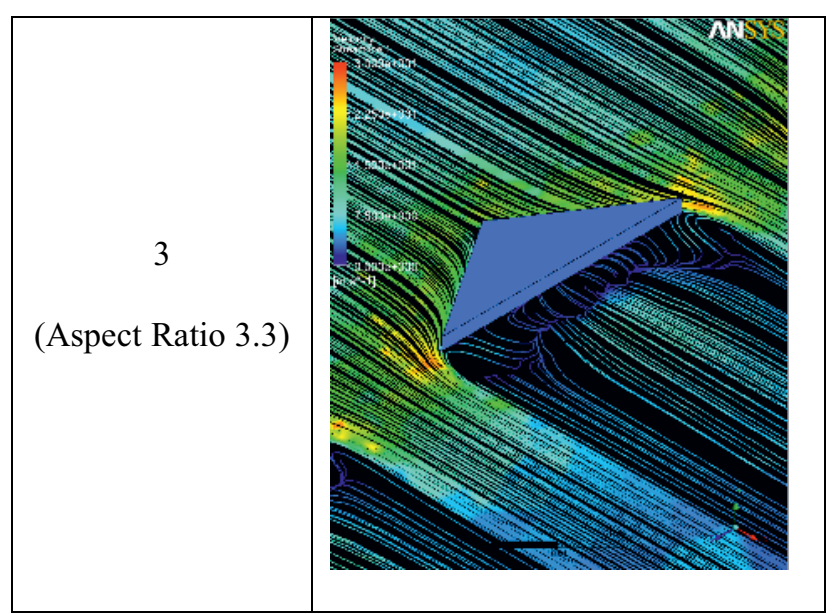

Table 2 shows surface velocity streamlines and vortices on the three triangular configurations. The behaviour of the vortices differs from a configuration to another. This phenomenon causes vacuum that sucks the free stream flow on to the surface, thus delaying flow separation. Furthermore as the width of triangles increase, the vortices magnitude and formation increase.

\subsection{Three configurations comparison}

Figures 22, 23 and 24 are provided to show a comparison between the velocity projections on the three micro vortex generators on the top view. According to the shown figure the flow is accelerated on the sides of two TMVGs with aspect ratio of 2.0 and 1.3 from $15 \mathrm{~m} / \mathrm{s}$ to $22.5 \mathrm{~m} / \mathrm{s}$, after that due to backward facing step there is a reduction in velocity on the edge of these two configurations. The quality of the generated vortex of these two configurations can be rated to be better for the TMVG with aspect ratio of 2.0 than the TMVG with aspect ratio of 1.3.

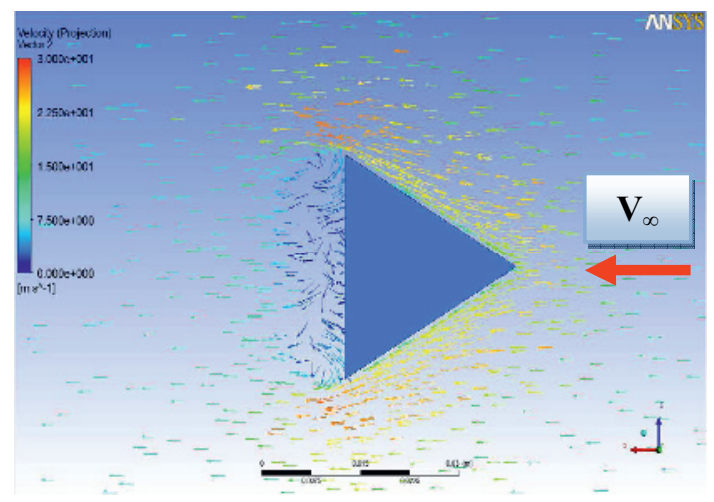

Figure 22. Micro Vortex Generator with Aspect Ratio 2.0 


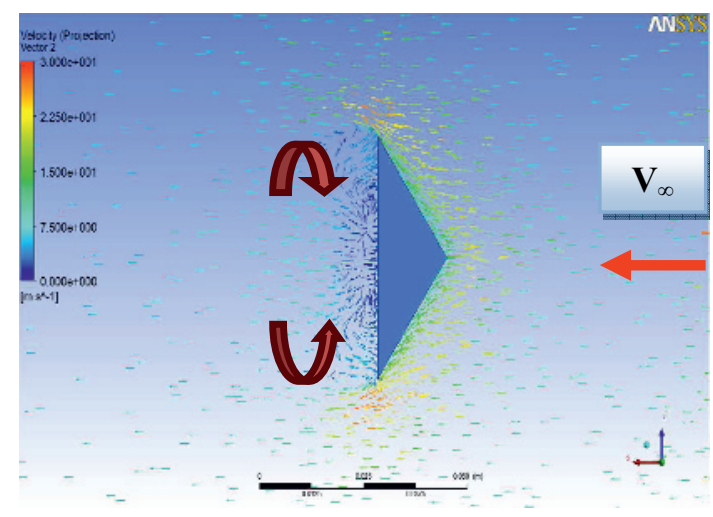

Figure 23. Micro Vortex Generator with Aspect Ratio 3.3

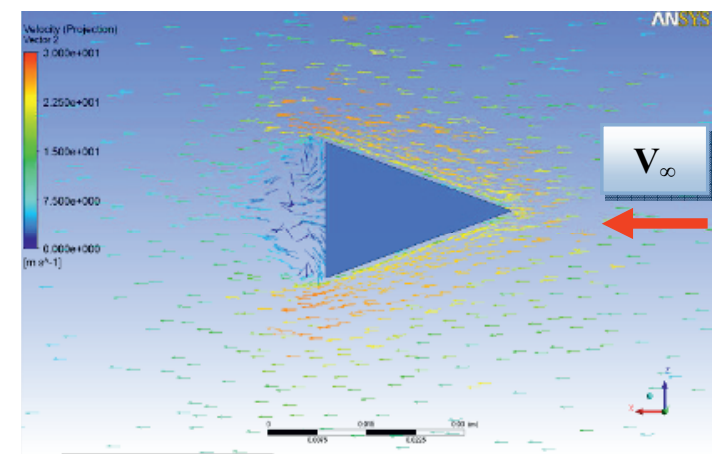

Figure 24. Micro Vortex Generator with Aspect Ratio 1.3

Unlike the other two vortices the TMVG with aspect ratio of 3.3 , shown in figure 23 , shows that the flow is decelerated on the sides from $22.5 \mathrm{~m} / \mathrm{s}$ to $15 \mathrm{~m} / \mathrm{s}$ because there is a sudden change in the angle and the airflow cannot follow the angled sides of the TMVG. The deceleration of flow is basically separation of the airflow on the side of the MVG which creates recirculating flow on the sides. Subsequently when the airflow reaches the edge of the TMVG there is reduction of velocity to 7.5 $\mathrm{m} / \mathrm{s}$ and this reduction of the velocity is mainly due the sudden high steep behind the MVG. It can be observe that the vortex core region of this configuration is located near the centre of the MVG.

Finally it can be concluded that the vortex created on the TMVG have counter-rotating structure which is shown in figure 23 . This configuration with aspect ratio of 3.3 gives the best result compare to other two lower aspect ratio configuration.

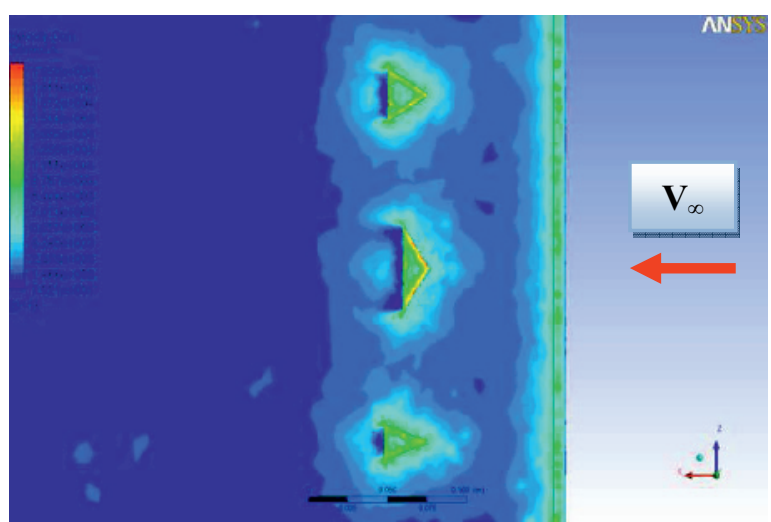

Figure 25. Velocity Curl top view

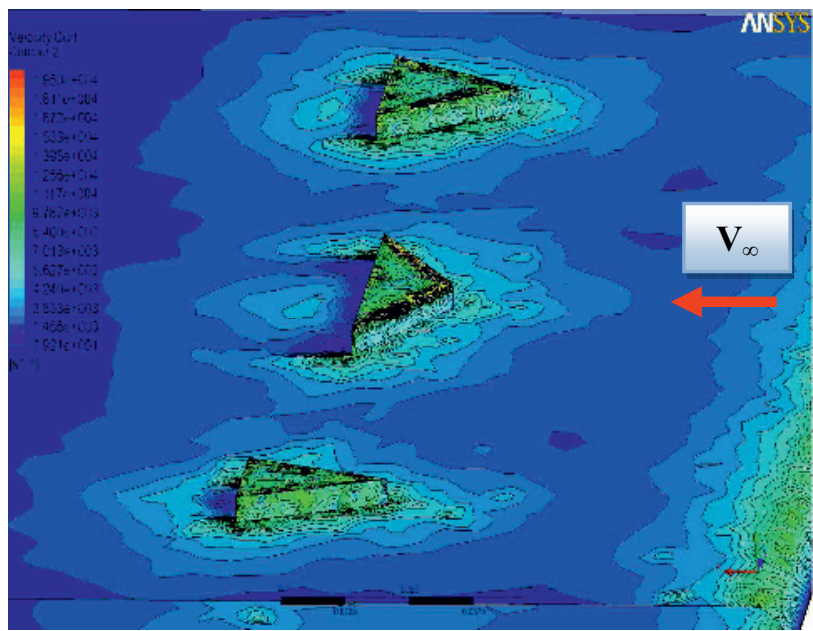

Figure 26. Velocity Curl isometric view

Figure 25 and 26 shows velocity curl contour on the three configurations, this figure is provided to illustrate the strength of vortex on different locations on top of the TMVGs. Generally velocity curl describes any microscopic rotation of velocity vectors and the value of velocity curl is representing circulation density.

It can be seen from shown figure that high velocity curl exist on the top surface of the leading edge of the MVG with aspect ratio of 3.3 and this velocity curl has lower strength for the other two configuration.

Velocity curl can be named as power of the circulation of the airflow or in another word the density of circulation. It shows that the MVG with aspect ratio of 3.3 creates more vortices than the other two configurations. Moreover it can be visualize that two kind of vortex is created by the MVG with aspect ratio of 3.3 in which the first type is due to backward facing step and the second type is due to the sudden change of angle of the MVG. 
Table 1. Velocity curl strength

\begin{tabular}{|c|c|}
\hline Aspect Ratio & $\begin{array}{c}\text { Velocity Curl } \\
\text { (Circulation density) }\end{array}$ \\
\hline 1.3 & $19500 \mathrm{~s}^{-1}$ \\
\hline 2.0 & $15330 \mathrm{~s}^{-1}$ \\
\hline 3.3 & $84000 \mathrm{~s}^{-1}$ \\
\hline
\end{tabular}

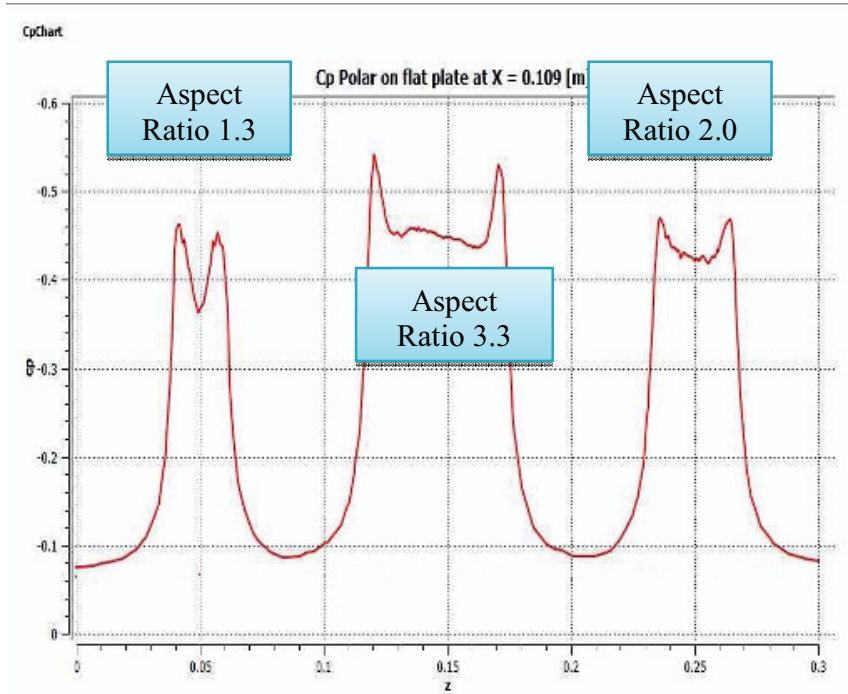

Figure 27, Spanwise pressure coefficient variation of three configurations with distance of $0.11 \mathrm{~m}$ from leading edge of flat plate

Pressure coefficient variation of three configurations behind three different configurations of MVGs is shown in this figure 27. The importance of this figure is in verifying the location of vortex core region by knowing that maximum pressure reduction will occur in the vortex core region. So it can be seen that in all three configurations vortex core region is located on the two rear sharp edges of MVGs.

Table 2. Minimum pressure coefficient

\begin{tabular}{|c|c|}
\hline Aspect Ratio & $\begin{array}{c}\text { Min Pressure } \\
\text { Coefficient }\end{array}$ \\
\hline 3.3 & $-0.52 \sim-0.55$ \\
\hline 2.0 & -0.44 \\
\hline 1.3 & -0.41 \\
\hline
\end{tabular}

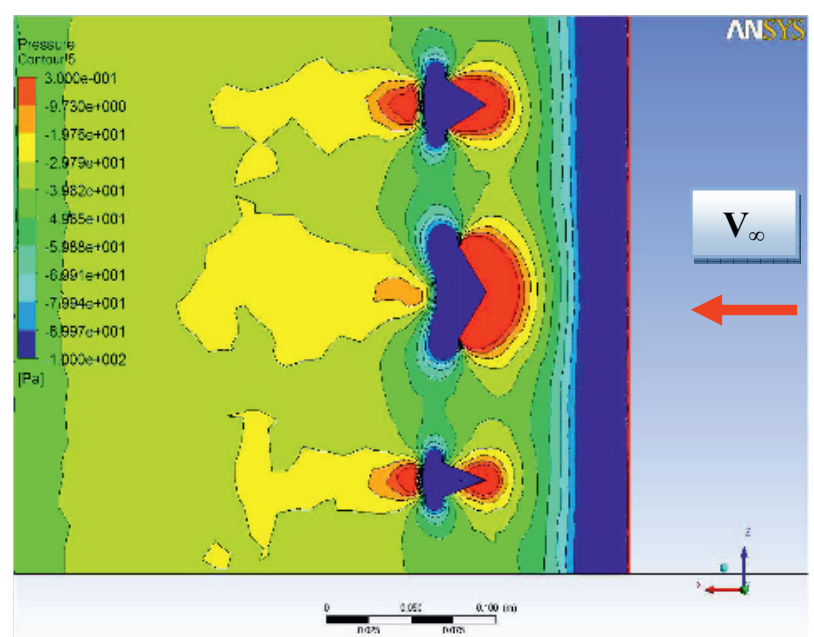

Figure 28. Pressure contour comparison

Pressure contour comparison between all three configurations is illustrated in figure 28 . It can be seen that maximum pressure occurs at the tip of the TMVGs due to stagnation point which have lower velocity resulting in having maximum pressure. And at the back of TMVGs there is a sudden pressure drop which occurs due to two main reasons of backward facing step and recirculating region. In circulating region at the core the lowest pressure is investigated and as circulation density decreases static pressure rises.

\section{Comparison between Theoretical and Analytical}

Figure 29 represents the experimental results along with the ANSYS computed results. The solid line represents the ANSYS results for pressure coefficient up till tap 20 which is $24 \mathrm{~cm}$ away from the leading edge, with each tap having an equal spacing of $1.2 \mathrm{~cm}$. The solid line represents the experimental results up till the same tap on the flat plate. It can be noted that the experimental results follow a similar path of that of ANSYS generated results. However the difference seen between the two results is due to finishing error of the surface (surface roughness) and human error in manufacturing of the holes for tapping.

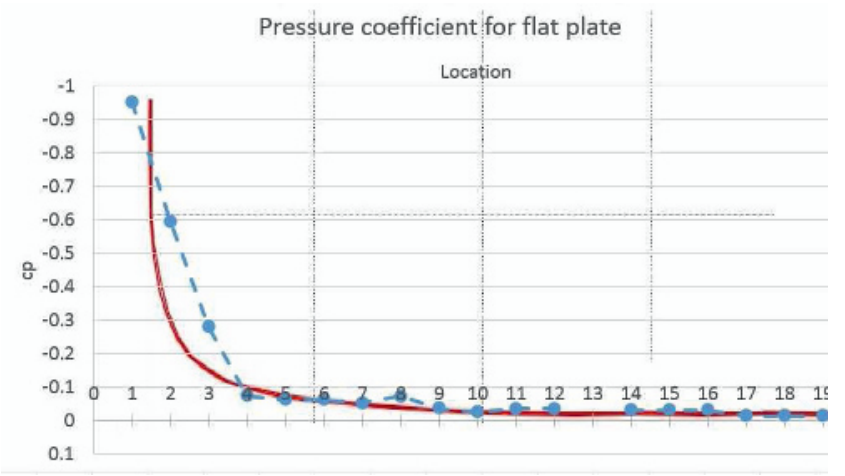

Figure 29. Comparison between experimental and Analytical pressure coefficient 


\section{Further studies}

By experimenting and performing CFD analysis on various configuration of the TMVGs it can be concluded that the Wide triangle with aspect ratio of 3.3 is capable of producing strong vortexes, compared to the other two configurations.

Moreover, during the flow visualization experiment on various TMVGs, it was observed a new axis of vortex being formed over the leading edge of the MGVs. Such behavior requires more research and experimentation on finding it effects on flow separation.

On a complete scale, the benefits of such TMVGs can only be proven effective once under experiments and analysis that can represent a wing or places of separation. Further development objectives are to investigate the effects of MGVs on flow separation by simulating it through experiments and CFD analysis in flow field of a wing.

\section{References}

1. D. Anderson, J. R. Fundamental of Aerodynamics, (1984)

2. A. Pierce, experimental study of micro-vortex generators, (2010)

3. K. Kusunose, N. J. Yu, Vortex generator installation drag on an airplane near its cruise condition (2003)

4. F. K. Lu, A. J. Pierce, Y. Shih, C. Liu, A. Li, Experimental and Numerical study of flow Topology past micro vortex generator (2010) 